\title{
THE STRUCTURE DISTRIBUTION IN A MIXED POISSON PROCESS
}

\author{
JOZEF L. TEUGELS and PETRA VYNCKIER \\ JLT, Department of Mathematics, \\ Katholieke Universiteit Leuven, \\ Celestijnenlaan 200B, B-3001, \\ Heverlee (Leuven), Belgium.
}

(Received July, 1996; Revised October, 1996)

\begin{abstract}
We use a variety of real inversion formulas to derive the structure distribution in a mixed Poisson process. These approaches should prove to be useful in
\end{abstract} applications, e.g., in insurance where such processes are very popular.

This article is dedicated to the memory of Roland L. Dobrushin.

Key words: Mixed Poisson Process, Inversion Formulas.

AMS (MOS) subject classifications: $60 \mathrm{G} 55$.

\section{Introduction}

One of the most classical examples of a counting process $\{N(t) ; t \geq 0\}$ is the homogeneous Poisson process. The probability distribution is given on $\mathbb{N}$ in the form:

$$
p_{n}(t)=P(N(t)=n)=\frac{e^{-\lambda t}(\lambda t)^{n}}{n !}
$$

The risk-parameter $\lambda$ gives the average number of events per unit time.

In many applications, however, the Poisson process is too simple to be applicable.

Example: If $N(t)$ is the number of claims up to time $t$ in a specific insurance portfolio, then it has been known to actuaries that the variability in the portfolio-expressed by $\operatorname{Var}\{N(t)\}$ - is much larger than $\lambda t$, the value corresponding to the strict Poisson case. One reason is that, even when the number of claims for each individual policy follows a Poisson distribution, the averages vary over the portfolio. This means that the value $\lambda$ for an individual policy is one of the possible values of a random variable $\Lambda$. This then leads to the notion of a mixed Poisson process, which is defined as follows (see Lundberg, [11]).

Definition: A mixed Poisson process (MPP) $\{N(t) ; t \geq 0\}$ is a pure birth process with state space $\mathbb{N}$ and counting distribution $p_{n}(t)$ of the form:

$$
p_{n}(t)=P(N(t)=n)=\int_{0}^{\infty} \frac{e^{-\lambda t}(\lambda t)^{n}}{n !} d H(\lambda),
$$

where $H$ is the structure distribution given by $H(\lambda)=P\{\Lambda \leq \lambda\}$ with $H(0)=0$.

Here are a few popular choices for the structure function. 
- Homogeneous Poisson process. The random variable $\Lambda$ is degenerate at $\lambda(>0)$. This is the only MPP that is simultaneously a renewal process. The interclaim times are independent and exponentially distributed.

- Double Poisson process. The structure distribution has two different jump points, $\lambda_{1}$ and $\lambda_{2}$, with corresponding heights, $p_{1} \in(0,1)$ and $p_{2}=1-p_{1}$, respectively. The counting distribution is then

$$
p_{n}(t)=p_{1} \frac{e^{-\lambda_{1} t}\left(\lambda_{1} t\right)^{n}}{n !}+p_{2} \frac{e^{-\lambda_{2} t}\left(\lambda_{2} t\right)^{n}}{n !}, t>0 .
$$

This kind of process might be used if the population were to be subdivided into females and males.

- Pólya or Pascal process. For this example, the structure distribution is $\operatorname{Gamma}(\alpha, 1 / b)$, where $\alpha, b>0$, so that

$$
d H(\lambda)=\frac{b^{\alpha}}{\Gamma(\alpha)} e^{-b \lambda} \lambda^{\alpha-1} d \lambda
$$

The resulting counting distribution is then

$$
p_{n}(t)=\left(\begin{array}{c}
\alpha+n-1 \\
n
\end{array}\right)\left(\frac{t}{t+b}\right)^{n}\left(\frac{b}{t+b}\right)^{\alpha}, t>0
$$

which is a Pascal or negative binomial distribution. The two parameters, $\alpha$ and $b$, allow great flexibility while one fits actual data to this theoretical distribution.

- $\quad$ Sichel process. In [12], Sichel introduced a distribution as a mixed Poisson by mixing it with a general inverse Gaussian distribution of the form:

$$
h(\lambda)=\frac{d H(\lambda)}{d \lambda}=\frac{\mu^{-\theta} \lambda^{\theta-1}}{2 K_{\theta}\left(\frac{\mu}{\beta}\right)} \exp \left\{-\frac{\lambda^{2}+\mu^{2}}{2 \beta \lambda}\right\},
$$

where the three parameters, $\beta, \theta$ and $\mu$, are nonnegative. The function $K_{\theta}$ is the modified Bessel function of the third kind. An explicit form of the probabilities can be obtained so that

$$
p_{n}(t)=\frac{(\mu t)^{n}}{n !}(1+2 \beta t)^{-\frac{1}{2}(\theta+n)} \frac{K_{\theta+n}\left(\frac{\mu}{\beta} \sqrt{1+2 \beta t}\right)}{K_{\theta}\left(\frac{\mu}{\beta}\right)} .
$$

The case where $\theta=-1 / 2$ is particularly interesting since the general inverse Gaussian distribution simplifies to the classical inverse Gaussian distribution.

The introduction of a general MPP is probably due to Thyrion [15] for the general case and to Ammeter [4] for the special case of the Pascal process. The first detailed and fundamental study of MPP's is due to Lundberg [11], who derived the deeper connection between MPP's and continuous-time Markov chains. In particular, Lundberg derived the binomial criterion as a characterization of MPP's among Markov processes.

Other contributions are due to Albrecht [1-3], who has been the first to discuss statistical problems connected with MPP's. For more information on MPP's, see Johnson and Kotz [9], Bühlmann[5], Gerber [6,7] and Grandell [8]. Moment estimators and maximum likelihood estimators for the structure distribution have been derived by Tucker [16] and Simar [13]; they result in discrete estimates for $H$. Again, Albrecht [3] studied estimators for the case of a mixture of a known finite number of Poisson components. In all these estimators, one uses the number of claims in successive repetitions of the process.

An alternative approach is due to Karr [10], who estimated $H$ by inverting the Laplace transform. In this case, only the time epoch of the first claim in each of the realizations of the MPP is used. Our approach is more in the spirit of Karr's. 


\section{Estimation of $H$ by Real Inversion Formulas}

Before we start deriving inversion formulas, let us introduce an abbreviation. For $0 \leq y_{1} \leq$ $y_{2}<\infty$, we shall write

$$
H\left\{y_{1} ; y_{2}\right\}:=\frac{1}{2} H\left(\left\{y_{1}\right\}\right)+H\left(y_{1}, y_{2}\right)+\frac{1}{2} H\left(\left\{y_{2}\right\}\right)
$$

In the above $H(\{y\})$ is the point mass of $H$ at the point $y$, while $H\left(y_{1}, y_{2}\right)$ denotes the mass in the open interval $\left(y_{1}, y_{2}\right)$.

\subsection{Inversion using the Laplace transform of $H$}

This method is based on the equality:

where $\widehat{H}$ is the Laplace transform of $H$.

$$
\widehat{H}(\theta)=E\left(\left(1-\frac{\theta}{t}\right)^{N(t)}\right)
$$

We know that

$$
\begin{aligned}
& E\left(\left(1-\frac{\theta}{t}\right)^{N(t)}\right)=\int_{0}^{\infty} e^{-\lambda t} \sum_{n=0}^{\infty} \frac{\left(\left(1-\frac{\theta}{t}\right) \lambda t\right)^{n}}{n !} d H(\lambda) \\
& =\int_{0}^{\infty} e^{-\lambda t} e^{\left(1-\frac{\theta}{t}\right) \lambda t} d H(\lambda)=\int_{0}^{\infty} e^{-\lambda \theta} d H(\lambda)=\widehat{H}(\theta) .
\end{aligned}
$$

\subsubsection{Inversion via Poisson variates}

The following limiting relation can be derived by looking at a sequence of independent, identically distributed Poisson variables. See, for example, Teugels [14].

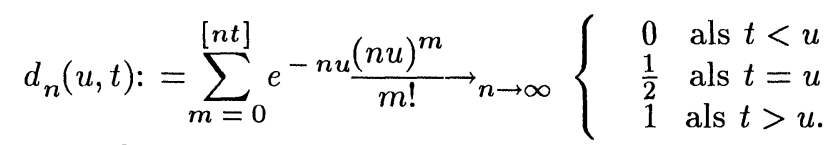

Define, for $0<y_{1}<y_{2}<\infty$, the expression:

$$
I_{n}\left(y_{1}, y_{2}\right):=\sum_{m=1+\left[n y_{1}\right]}^{\left[n y_{2}\right]} \frac{(-n)^{m}}{m !} \widehat{H}^{(m)}(n)
$$

Using the definition of $\widehat{H}$, we can write that

$$
\begin{aligned}
I_{n}\left(y_{1}, y_{2}\right) & =\sum_{m}^{\left[n y_{2}\right]} \frac{(-n)^{m}}{m !}(-1)^{m} \int_{0}^{\infty} e^{-\lambda n} \lambda^{m} d H(\lambda) \\
= & \int_{0}^{\infty}\left(\sum_{m=1+\left[n y_{1}\right]}^{\left[n y_{2}\right]} \frac{(n \lambda)^{m}}{m !} e^{-\lambda n}\right) d H(\lambda) \\
& =\int_{0}^{\infty}\left\{d_{n}\left(\lambda, y_{2}\right)-d_{n}\left(\lambda, y_{1}\right)\right\} d H(\lambda) .
\end{aligned}
$$

On the other hand, equality (2) can be invoked to write

$$
\widehat{H}^{(m)}(\theta)=(-t)^{-m} E\left\{\frac{N(t) !}{(N(t)-m) !}\left(1-\frac{\theta}{t}\right)^{N(t)-m}\right\} .
$$


Interchanging summation and expectation, we see that

$$
I_{n}\left(y_{1}, y_{2}\right)=E\left\{\sum_{m=1+\left[n y_{1}\right]}^{\left[n y_{2}\right]}\left(\begin{array}{c}
N(t) \\
m
\end{array}\right)\left(\frac{n}{t}\right)^{m}\left(1-\frac{n}{t}\right)^{N(t)-m}\right\} .
$$

We combine this with the first formula and apply Lebesgue's dominated convergence theorem to arrive at a first inversion formula:

$$
H\left\{y_{1}, y_{2}\right\}=\lim _{n \uparrow \infty} E\left\{\sum_{m=1+\left[n y_{1}\right]}^{\left[n y_{2}\right]}\left(\begin{array}{c}
N(t) \\
m
\end{array}\right)\left(\frac{n}{t}\right)^{m}\left(1-\frac{n}{t}\right)^{N(t)-m}\right\} .
$$

For the mass at a point, we can use a slightly different approach than that for $n \rightarrow \infty$ :

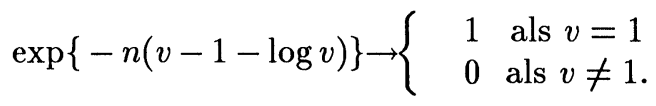

Following the same argument as above, we get

$$
H(\{y\})=\lim _{n \uparrow \infty} E\left\{\frac{N(t) !}{(N(t)-n) !}\left(1-\frac{n}{t y}\right)^{N(t)-n}\left(\frac{e}{t y}\right)^{n}\right\} .
$$

\subsubsection{Inversion via Gamma variates}

An analogous derivation can be made starting from a sequence of i.i.d. exponential variables. For the latter we have the limiting relation:

Define

$$
e_{n}(u):=\frac{1}{\Gamma(n)} \int_{0}^{n u} e^{-v} v^{n-1} d v \rightarrow_{n \rightarrow \infty}\left\{\begin{array}{lll}
0 & \text { als } u<1 \\
\frac{1}{2} & \text { als } u=1 \\
1 & \text { als } u>1
\end{array}\right.
$$

$$
J_{n}\left(y_{1}, y_{2}\right):=\int_{y_{1}}^{y_{2}} \frac{(-n)^{n}}{\Gamma(n)} \widehat{H}^{(n)}\left(\frac{n}{s}\right) \frac{d s}{s^{n+1}}
$$

We easily find from the definition of $\widehat{H}$ that

From (2) we see that

$$
J_{n}\left(y_{1}, y_{2}\right)=\int_{0}^{\infty}\left\{e_{n}\left(\frac{\lambda}{y_{1}}\right)-e_{n}\left(\frac{\lambda}{y_{2}}\right)\right\} d H(\lambda)
$$

$$
J_{n}\left(y_{1}, y_{2}\right)=E\left\{\frac{1}{B(n, N(t)-n+1)} \int_{\frac{n}{t y_{2}}}^{\frac{n}{t y_{1}}} z^{n-1}(1-z)^{N(t)-n} d z\right\} .
$$

Combining these elements with Lebesgue's theorem, we obtain a second inversion formula:

$$
H\left\{y_{1} ; y_{2}\right\}=\lim _{n \uparrow \infty} E\left\{\frac{1}{B(n, N(t)-n+1)} \int_{\frac{n}{t y_{2}}}^{\frac{n}{t y_{1}}} z^{n-1}(1-z)^{N(t)-n} d z\right\} .
$$

One advantage of this latter formula is that, if the density $h$ of $H$ exists, then

$$
h(y)=\lim _{n \uparrow \infty} E\left\{\frac{1}{B(n, N(t)-n+1)} \frac{1}{y}\left(\frac{n}{y t}\right)^{n}\left(1-\frac{n}{y t}\right)^{N(t)-n}\right\} .
$$




\subsubsection{Normal approximation}

For both of the inversion formulas, (4) and (6), one can apply a normal approximation. Let us illustrate the procedure on (6).

In (6), let $z=a_{n} u+b_{n}$, where $a_{n}$ and $b_{n}$ are functions of $t$ that are to be determined in the sequel. Rewrite the incomplete beta-integral in the form:

$$
\int_{\frac{n}{t y_{2}}}^{\frac{n}{t y_{1}}} z^{n-1}(1-z)^{N(t)-n} d z=a_{n} \int_{\alpha_{2}(n)}^{\alpha_{1}(n)}\left(a_{n} u+b_{n}\right)^{n-1}\left(\bar{b}_{n}-a_{n} u\right)^{N(t)-n} d u,
$$

with $\bar{b}_{n}=1-b_{n}$ and

$$
\alpha_{i}(n)=\frac{1}{a_{n}}\left(\frac{n}{t y_{i}}-b_{n}\right), \quad i=1,2 .
$$

The expression inside the expectation sign in (6) then reads as

$$
\frac{1}{B(n, N(t)-n+1)} \int_{\frac{n}{t y_{2}}}^{\frac{n}{t y_{1}}} z^{n-1}(1-z)^{N(t)-n} d z=I_{1}(n) \int_{\alpha_{2}(n)}^{\alpha_{1}(n)} \exp \left(I_{2}(n, u)\right) d u,
$$

where

$$
I_{1}(n)=\frac{a_{n} b_{n}^{n-1} \bar{b}_{n}^{N(t)-n} N(t) !}{(n-1) !(N(t)-n) !}
$$

and

$$
I_{2}(n, u)=(n-1) \log \left(1+\frac{a_{n}}{b_{n}} u\right)+(N(t)-n) \log \left(1-\frac{a_{n}}{\bar{b}_{n}} u\right)
$$

Now choose $a_{n}$ and $b_{n}$ such that $\exp \left(I_{2}(n, u)\right)$ converges to the key factor in the normal density. We then need both $N(t)$ and $n$ to be large, but also $N(t)-n$ needs to be large. A series expansion of the logarithms yields for $I_{2}$ that

$$
I_{2}(n, u)=u a_{n}\left\{\frac{n-1}{b_{n}}-\frac{N(t)-n}{\bar{b}_{n}}\right\}-\frac{u^{2}}{2} a_{n}^{2}\left\{\frac{n-1}{b_{n}^{2}}+\frac{N(t)-n}{\bar{b}_{n}^{2}}\right\}+O\left(\frac{a_{n}}{b_{n}} u\right)^{3} .
$$

The obvious choice for $b_{n}$ should annihilate the first term on the right. The subsequent choice of $a_{n}$ is made to reduce the coefficient of $-u^{2} / 2$ to 1 . This yields the choices:

$$
b_{n}=\frac{n-1}{N(t)-1}, \bar{b}_{n}=\frac{N(t)-n}{N(t)-1}, \text { and } a_{n}^{2}=\frac{(n-1)(N(t)-n)}{(N(t)-1)^{3}} .
$$

With the help of these expressions and Stirling's formula, one easily shows that $I_{1}(n) \sim(2 \pi)^{-\frac{1}{2}}$. Introducing the above values for $a_{n}$ and $b_{n}$ in $\alpha_{i}$ yields

$$
\alpha_{i}(n)=\frac{(N(t)-1)^{\frac{3}{2}}}{\sqrt{(n-1)(N(t)-n)}}\left\{\frac{n}{t y_{i}}-\frac{n-1}{N(t)-1}\right\} \sim \sqrt{\frac{n}{1-\frac{n}{N(t)}}}\left\{\frac{N(t)}{t y_{i}}-1\right\} .
$$

Combining all of the above results, one obtains a normal approximation to (6):

$$
H\left\{y_{1} ; y_{2}\right\} \sim \Phi\left\{\sqrt{\frac{n}{1-\frac{n}{N(t)}}}\left(\frac{N(t)}{t y_{1}}-1\right)\right\}-\Phi\left\{\sqrt{\frac{n}{1-\frac{n}{N(t)}}}\left(\frac{N(t)}{t y_{2}}-1\right)\right\} .
$$

\subsection{Inversion based on the time epochs}

As an alternative, we can start from the explicit expression for the distribution of the epoch of the $n$th event, $T_{n}$, and combine this with the limiting relation (5). According to a result by 
Lundberg [11], (see alternatively, Albrecht [3]),

$$
f_{T_{n}}(x)=\frac{n}{x} p_{n}(x)=\int_{0}^{\infty} e^{-\lambda x} \lambda^{n} \frac{x^{n-1}}{(n-1) !} d H(\lambda) .
$$

For the distribution we get

Henceforth,

$$
\int_{0}^{w} f_{T_{n}}(u) d u=\int_{0}^{\infty} d H(\lambda) \int_{0}^{\lambda w} e^{-v} \frac{v^{n-1}}{(n-1) !} d v=\int_{0}^{\infty} e_{n}\left(\frac{\lambda w}{n}\right) d H(\lambda) .
$$

$$
\begin{aligned}
P\left(y_{1}\right. & \left.\leq \frac{n}{T_{n}}<y_{2}\right)=P\left(T_{n} \leq \frac{n}{y_{1}}\right)-P\left(T_{n} \leq \frac{n}{y_{2}}\right) \\
& =\int_{0}^{\infty}\left\{e_{n}\left(\frac{\lambda}{y_{1}}\right)-e_{n}\left(\frac{\lambda}{y_{2}}\right)\right\} d H(\lambda) .
\end{aligned}
$$

Now, we apply (5) directly to obtain a fourth inversion formula:

$$
H\left\{y_{1} ; y_{2}\right\}=\lim _{n \uparrow \infty} P\left(y_{1} \leq \frac{n}{T_{n}}<y_{2}\right) .
$$

\section{Simulations}

\subsection{Simulation of an MPP}

Another important property of an MPP is that the waiting times between epochs of events, with each $W_{n}=T_{n-1}-T_{n}$, are dependent, unless we are dealing with a strict Poisson process. Albrecht [3] has shown that the $W_{n}$ 's are identically distributed, exchangeable, but positively correlated. More specifically, one has

$$
\operatorname{Cov}\left(W_{n-1}, W_{n}\right)=\operatorname{Var}\left(\frac{1}{\Lambda}\right)
$$

There are at least two distinct procedures to simulate the time epochs of an MPP.

(i) A first method to simulate the time epochs of an MPP uses the above interdependence between the waiting times. Recall from Albrecht [3] that

$$
P\left(W_{n} \leq t \mid T_{n-1}=t_{n-1}\right)=1-\left(\frac{t_{n-1}}{t+t_{n-1}}\right)^{n-1} \frac{p_{n-1}\left(t+t_{n-1}\right)}{p_{n-1}\left(t_{n-1}\right)} .
$$

(ii) A second method is based on the uniformity property of the MPP. Given that $N(t)=n$, the first $n$ epochs, $T_{1}, \ldots, T_{n}$, have the same joint distribution as the order statistics of a sample from a uniform distribution on $(0, t)$ (Albrecht [3]). One starts with the simulation of $n$ as a value of $N(t)$ with a given value of $t$ and in accordance with the distribution $p_{n}(t)$. One then simulates $n$ random numbers in $(0, t)$. After rearrangement, these values give a sample $\left(T_{1}, \ldots, T_{n}\right)$.

\subsection{Illustrations}

We perform simulations for the empirical versions of the inversion formulas given in (4), (7) and (8) on homogeneous, double Poisson processes, Pascal processes, and mixtures of Pascal processes with Poisson point masses. In all cases, we considered the distribution function by taking $y_{1}$ to be zero. From the simulations, we can conclude that the estimators for the inversions (4) and (8) perform quite well, even for a relatively small number of realizations. On the other hand, the normal approximation in (7) seems to be too rough. Generally, continuous structure functions are better estimated by the inversion formulas than discrete distributions are. By means of example, the results of two simulations are shown in Figures (a) and (b). 
(a)

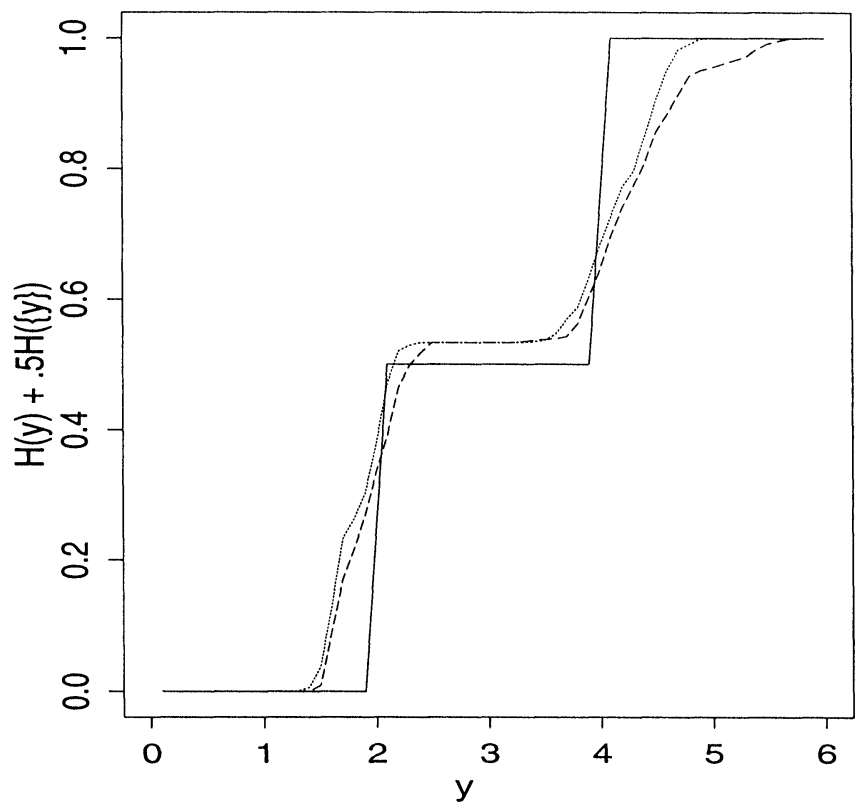

Figure (a)

(b)

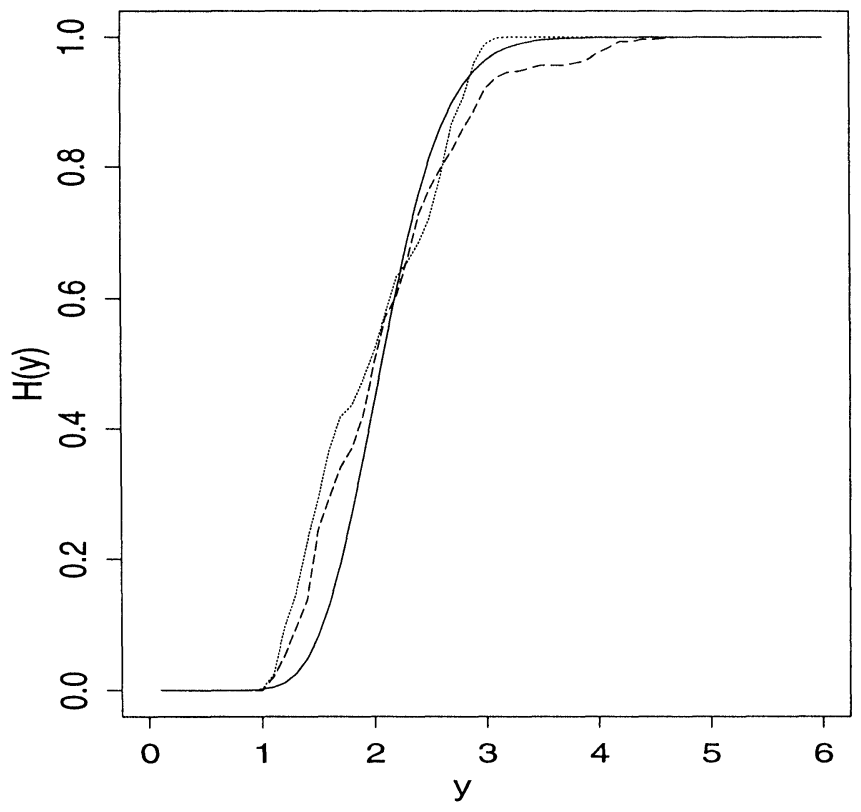

Figure (b) 
In both parts, the estimators are based on 15 simulated realizations with fixed $t=20$. The solid lines represent the theoretical structure function and the dotted lines represent the estimator for (8), while the points are the estimators for inversion formula (4). For Figure (a), we simulated double Poisson processes with parameters $\lambda_{1}=2, \lambda_{2}=4$ and $p_{1}=.5$. The value of $n$ in (4) was taken to be 20 . In the case of the estimator for (8), we considered the averages for $n$-values from $n=18$ to $n=N(20)$. Figure (b) concerns simulations of Pascal processes with parameters $a=21$ and $b=10$. The value for $n$ in (4) was taken to be 18 , whereas for (8) the averages for $n=15$ to $n=N(20)$ were considered.

\section{Acknowledgements}

The authors take pleasure in acknowledging D. Wauters for helping with simulations. They also enjoyed discussions with B. Sundt and H. Rootzén on the topic on mixed Poisson processes.

\section{References}

[1] Albrecht, P., Über einige Eigenschaften des gemischten Poisson prozesses, Bull. of the Assoc. of Swiss Actuaries (1981), 241-249.

[2] Albrecht, P., Zur statistischen analyse des gemischten Poisson-prozesses, gestützt auf Schadeneintrittzeitpunkte, Blätter der Deutschen Gesellschaft für Versicherungsmathematik 15 (1982), 249-257.

[3] Albrecht, P., On some statistical methods connected with the mixed Poisson process, Scand. Actuarial Journal (1982), 1-14.

[4] Ammeter, H., A generalization of the collective theory of risk in regard to fluctuating basic probabilities, Skand. Akt. 31 (1948), 171-198.

[5] Bühlmann, H., Mathematical Methods in Risk Theory, Springer-Verlag, Heidelberg 1970.

[6] Gerber, H.U., An Introduction to Mathematical Risk Theory, Hübner Foundation, University of Pennsylvania, Philadelphia 1979.

[7] Gerber, H., On the asymptotic behavior of the mixed Poisson process, Scand. Actuarial J. (1983), 256.

[8] Grandell, J., Aspects of Risk Theory, Springer-Verlag, New York 1991.

[9] Johnson, N.I. and Kotz, S., Mixed Poisson process, Encyclopedia of Statistical Sciences 5 (1985), 556-559.

[10] Karr, A.F., Combined nonparametric inference and state estimation for mixed Poisson processes, Zeitschrift für Wahrscheinlichkeitstheorie und verwandte Gebiete 66 (1984), 81-96.

[11] Lundberg, O., On Random Processes and their Application to Sickness and Accident Statistics, Almquist and Wicksells, Uppsala 1964.

[12] Sichel, H., On a family of discrete distributions particularly suited to represent long tailed frequency data, Proc. 3rd Symp. Math. Statistics (1971), Pretoria, CSIR.

[13] Simar, L., Maximum likelihood estimation of a compound Poisson process, The Annals of Statistics 4 (1976), 1200-1209.

[14] Teugels, J.L., Probabilistic proofs of some real inversion formulas, Math. Nachrichten 146 (1990), 149-157.

[15] Thyrion, P., Sur une propriété des processus de Poisson Généralisés, Bull. Assoc. Royale Actuaire Belges 59 (1959), 35-46.

[16] Tucker, H.G., An estimate of the compounding distribution of a compound Poisson distribution, Th. Prob. Appl. 8 (1963), 195-200. 


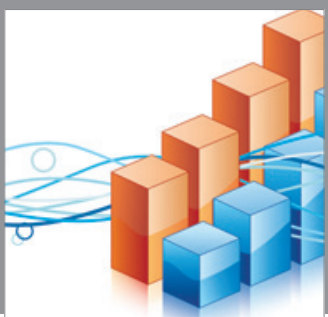

Advances in

Operations Research

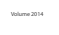

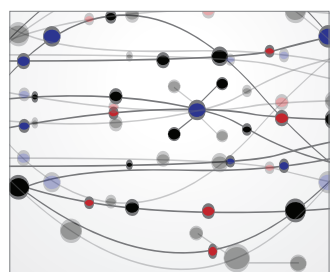

\section{The Scientific} World Journal
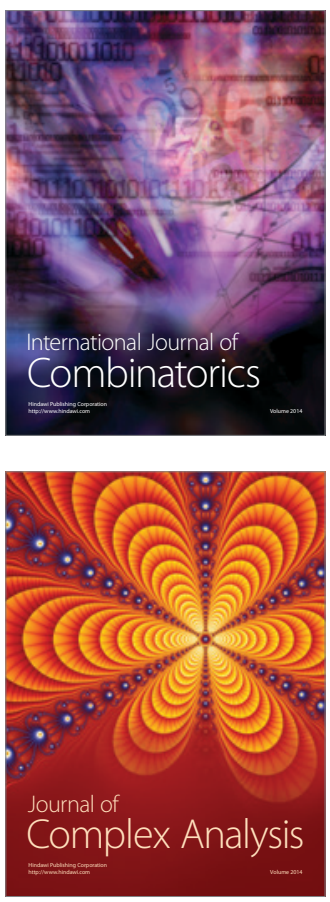

International Journal of

Mathematics and

Mathematical

Sciences
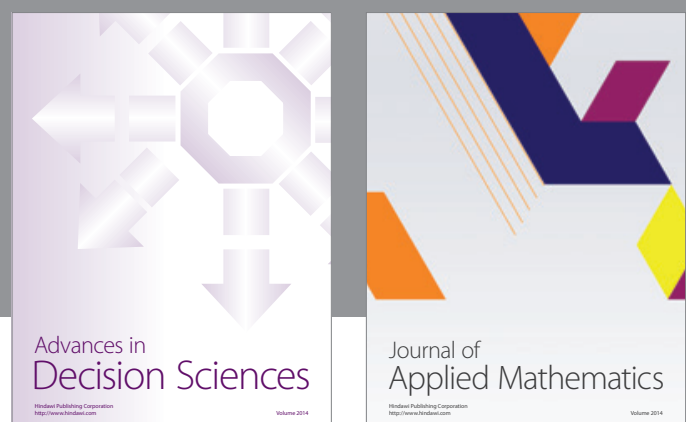

Journal of

Applied Mathematics
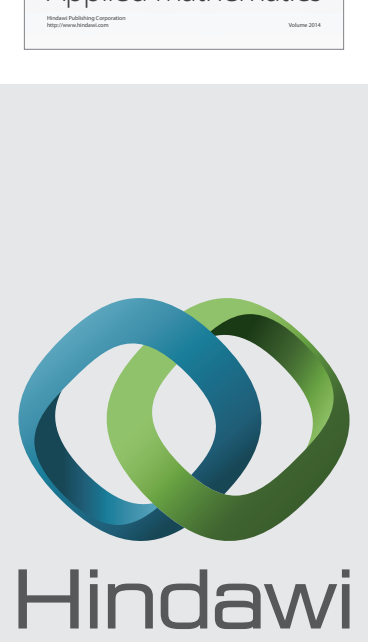

Submit your manuscripts at http://www.hindawi.com
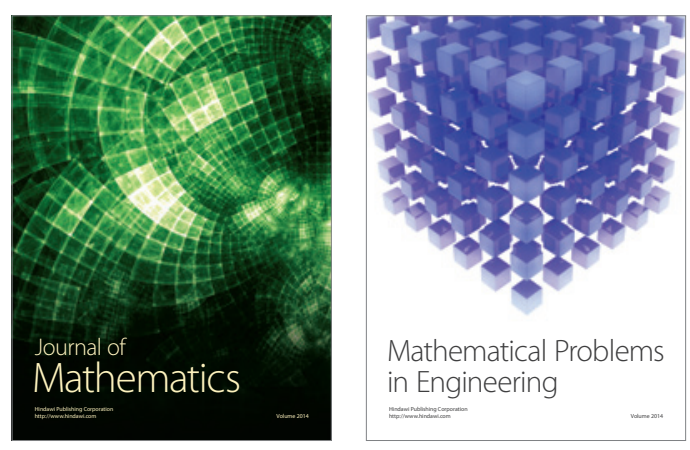

Mathematical Problems in Engineering
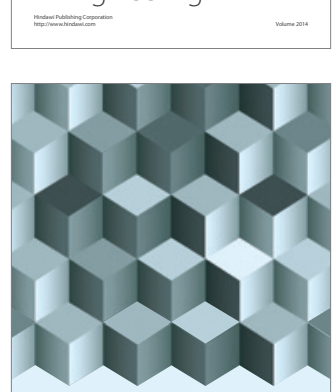

Journal of

Function Spaces
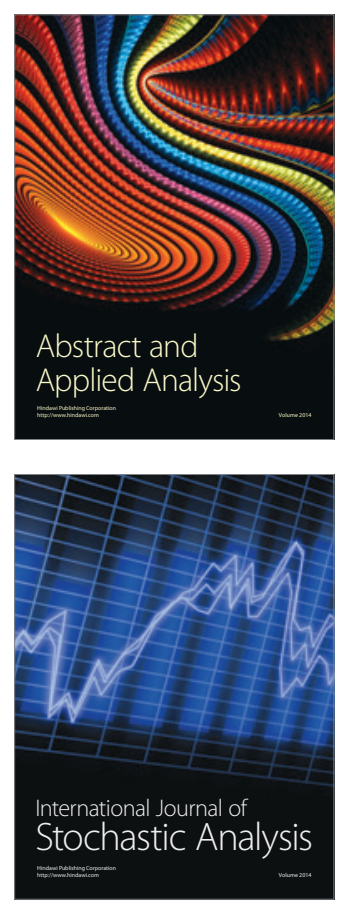

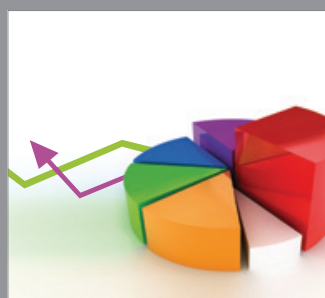

ournal of

Probability and Statistics

Promensencen
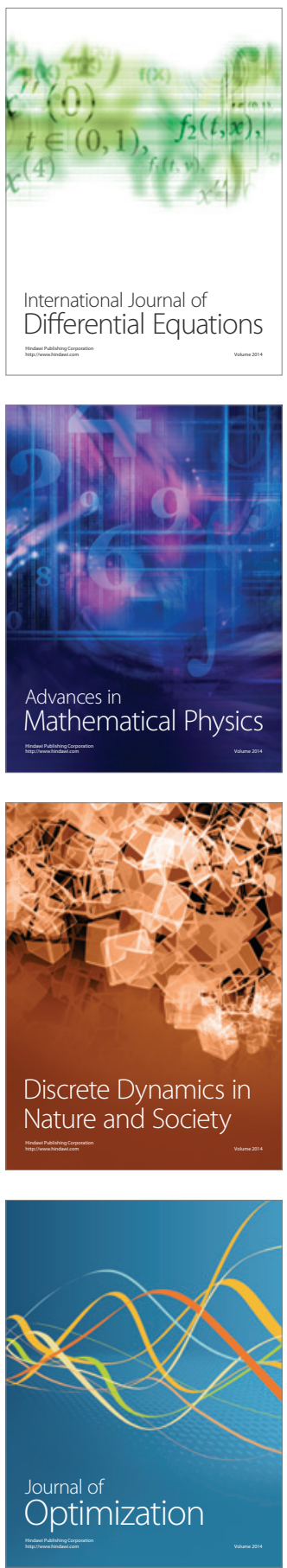